\title{
Coronary sinus diverticulum: importance, function, and treatment
}

\author{
Iman Razeghian-Jahromi ${ }^{1}$, Mohammad Nikoo ${ }^{1}$, and Andrea Natale ${ }^{2}$ \\ ${ }^{1}$ Shiraz University of Medical Sciences \\ ${ }^{2}$ St. David's Medical Center
}

June 17, 2020

\begin{abstract}
The importance of venous structure in the heart is usually overshadowed by that of the arterial system. Coronary sinus is a part of cardiac venous apparatus and connects left atria to the right atria. Other than having role in physiological contractions of both atria, it contributes substantially to cardiac electrical conduction system. Due to unique placement and connections of the CS, it has become growing interest in clinical cardiology. It is used in cardiac resynchronization therapy with biventricular pacing, left-sided catheter ablation of arrhythmias and administration of retrograde cardioplegia in cardiac surgery. In some individuals, CS is presented with anatomical variants. CS diverticulum is a congenital outpouching that provides muscular connection between atria and ventricle. This connection provides a suitable substrate for occurrence of arrhythmias, which even results in life-threatening events such as sudden cardiac death. Early diagnosis leads to treatment with ablation techniques which ultimately eliminates origins of arrhythmias.
\end{abstract}

Coronary sinus diverticulum: importance, function, and treatment

Iman Razeghian-Jahromi ${ }^{1 *}$, PhD, Mohammad Hossein Nikoo, MD ${ }^{1,2}$, Andrea Natale ${ }^{3,4,5,6,7,8}$

${ }^{1}$ Cardiovascular research center, Shiraz University of Medical Sciences, Shiraz, Iran

${ }^{2}$ Non-Communicable Diseases Research Center, Shiraz University of Medical Sciences, Shiraz, Iran

${ }^{3}$ Texas Cardiac Arrhythmia Institute, St. David's Medical Center, Austin, TX, USA; ${ }^{4}$ Dell Medical School, University of Texas, Austin, TX, USA; ${ }^{5}$ Department of Biomedical Engineering, University of Texas, Austin, TX, USA $;{ }^{6}$ MetroHealth Medical Center, Case

Western Reserve University School of Medicine, Cleveland, OH, USA $;{ }^{7}$ Division of Cardiology, Stanford University, Stanford, CA, USA; and ${ }^{8}$ Electrophysiology and Arrhythmia Services, California Pacific Medical Center, San Francisco, CA, USA

*Corresponding author: Iman Razeghian-Jahromi

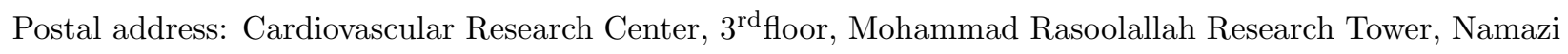
Hospital, Shiraz University of Medical Sciences, Shiraz, Iran.

Postal code: 71936-35899

Tel: +98 7136281561

Fax: +98 7136122235

Email: razejahromi@yahoo.com

Acknowledgements: N/A

Competing interests: The authors declare that they have no competing interests. 
Funding: This research was supported by Vice Chancellor of Research of Shiraz University of Medical Sciences.

Abstract

The importance of venous structure in the heart is usually overshadowed by that of the arterial system. Coronary sinus is a part of cardiac venous apparatus and connects left atria to the right atria. Other than having role in physiological contractions of both atria, it contributes substantially to cardiac electrical conduction system. Due to unique placement and connections of the CS, it has become growing interest in clinical cardiology. It is used in cardiac resynchronization therapy with biventricular pacing, left-sided catheter ablation of arrhythmias and administration of retrograde cardioplegia in cardiac surgery. In some individuals, CS is presented with anatomical variants. CS diverticulum is a congenital outpouching that provides muscular connection between atria and ventricle. This connection provides a suitable substrate for occurrence of arrhythmias, which even results in life-threatening events such as sudden cardiac death. Early diagnosis leads to treatment with ablation techniques which ultimately eliminates origins of arrhythmias.

Keywords: coronary sinus diverticulum; arrhythmia; ablation

Introduction

Coronary sinus (CS) anomalies such as diverticulum, persistent left superior vena cava or CS ostium dilatation are predominantly found in patients with accessory pathway-related tachycardias (1). Earlier studies have reported diverticulum of proximal CS in $7-11 \%$ of patients with posteroseptal accessory pathways (2). In this review, a literature appraisal was performed regarding importance, function, and ablation of CS diverticulum.

\section{CS}

Several cardiac procedures like targeted drug delivery, stem cell therapy as well as mapping and ablation of arrhythmias make use of CS (3). By a multipurpose catheter and injection of contrast in LAO and RAO views, CS anatomy was delineated. In the case of difficulty with catheter to cannulate, a deflectable sheath is employed (4). The average length ranges from 45 to $63 \mathrm{~mm}$. Different size of ostium ranges (4*5 to $9 * 16 \mathrm{~mm})$ have also been reported (5).

CS drains $60 \%$ of cardiac venous blood into the right atrium. In addition to the largest cardiac venous structure, it is an interatrial connection (6). However, unlike the veins, it has myocardial tissue which is the remnant of left atrial muscles (7). Although CS has been regarded as a venous structure for a long time but recently it was clarified its crucial role in the pathophysiology of atrial tachyarrhythmia (8-10).

The most common atrial arrhythmia in humans is atrial fibrillation (AF). Although pulmonary veins are closely associated to AF pathogenesis, it seems that ablation of these veins is not enough for disappearance of persistent AF. Additional reentry mechanism, probably by the involvement of CS was proposed (6). Muscular sleeve, which is around the proximal 25-50mm of the CS length, connects the two atria (11). Arrhythmia most likely emerges from these connections (12). Also, it was shown that CS has the capability for spontaneous depolarization and slow conduction, an indicator of intrinsic automaticity (13-15). Thereby, spontaneous firing of the cells in the CS is in charge for triggering, maintenance, and recurrence of AF $(12,16,17)$. Accordingly, ablation of CS together with pulmonary vein resulted in long term improvement through decreasing the recurrences (18).

\section{CS diverticulum}

Variation in CS anatomy in terms of position, length, and diameter was seen among individuals (19). CS anomalies are, but not limited to, enlargement, absence, atresia of right ostium, and hypoplasia (6). These anomalies may affect cardiac hemodynamics, which leads to clinical manifestations and requiring prompt diagnosis and treatment. However, some others are clinically occult without any obvious symptoms. They all have the potential to increase morbidity and mortality after certain surgical procedures. Moreover, electrophysiological characteristics of the CS are influenced by the existence of these anomalies $(20,21)$. 
CS diverticulum is a one of such anomalies. It is a CS congenital outpouching with a distinct neck that extends behind the left ventricle $(22,23)$. In most cases, diverticula were seen as a saccular dilatation and sometimes it is a fusiform dilatation. In some patients, its sac was bilobed. Contractility is its inherent characteristic (4). Diverticula are commonly found at the inferior aspect of the CS at its junction with the middle cardiac vein, 0 to $18 \mathrm{~mm}$ away from the CS ostium. The neck of the CS diverticulum is 5 to $10-\mathrm{mm}$ wide, opens into the CS and is proximal to the midcardiac vein (24). The orifice of the diverticula ranges between 2.6 to $15 \mathrm{~mm}$. The smallest and largest surface area was $0.73 \mathrm{~cm}^{2}$ and $6.45 \mathrm{~cm}^{2}$, respectively. The mean diameter of the CS was $7.05 \mathrm{~mm} \pm 1.90$. Thus, CS could be dilated up to $11 \mathrm{~mm}$ at measuring $3 \mathrm{~cm}$ proximal to the CS ostium (25, 26). (Fig. 1)

Central parts of cardiac conduction system including atrioventricular node and the His bundle are in the septal area. Approximately, one third of accessory pathways (APs) are located at this complex anatomic region. These pathways frequently have short refractory periods that predispose to rapid ventricular response during atrial fibrillation, thus increasing the risk of sudden death (24).

Therefore, precise localization of these pathways is critical during ablation of APs. APs of this area are classified into anteroseptal, midseptal, and posteroseptal (27). Diverticula with posteroseptal APs were first reported in 1985 (28). This type of AP increases the incidence of inducible AF (27). Myocardial fibers of CS diverticulum establish a connection between CS coat and the ventricle, which facilitates the incidence of AP $(29,30)$. Close proximity of the wall and the neck of the diverticula with posteroseptal and left posterior APs endangers patients to arrhythmias and even sudden cardiac death $(22,23)$.

Among 480 patients with posteroseptal or left posterior AP, CS diverticula was found in only 36 (7.5\%) (30). It is a rare anomaly which often diagnosed during venography and catheter ablation (31). The existence of CS diverticula is expected in the case of previous failed ablation, documented AF, when the best location for ablation is at the proximal of CS, and in specific ECG findings like polarity of the delta wave. A negative delta wave in lead II is an indicator of CS diverticulum with highest specificity and sensitivity $(29,30,32$, 33). (Fig 2.)

Treatment

Radiofrequency (RF) ablation is safe and treatment of choice for resolving septal APs (28). Percutaneous access for ablation of epicardial APs is feasible through CS cannulation. This was performed even in patients with previously failed endocardial ablation (34). Ablation of the CS eliminated focal tachyarrhythmia with CS origin (6). As CS has anatomic and electropgysiological relationship with atria, origin of multifocal tachyarrhythmia from either atria could be determined. If myocardial part of the CS is the origin, highfrequency higher-amplitude component indicates a near-field potential. Otherwise, (low-frequency loweramplitude) shows a far-field potential $(35,36)$.

Epicardial and endocardial ablation of the CS increase AF cycle length and eventually leads to AF termination (8). One study reported posteroseptal AP in a patient with a history of three previous failed attempts of ablation. In such cases, open chest surgical ablation is deemed as the only remaining choice. This difficultto-ablate AP was managed by epicardial mapping through pericardial catheter. Although, existence of high impedance due to thick epicardial fat hampered the ablation by this approach but a linear ablation within the CS targeting the earliest activation area terminated the preexcitation (37).

CS ablation is performed in patients with AF without previous pulmonary vein ablation (38). The anomalies of the CS sometimes becomes the crucial sites for successful ablation of posteroseptal pathways (1). Findings showed that CS diverticulum with APs were more prone to sudden death during AF due to acceleration of atrioventricular conduction (33). However, CS diverticulum is a well-known cause of unsuccessful AP ablation in wolff-parkinson-white syndrome. This fact underscores the necessity of identification and reporting of CS anomalies especially that of diverticula (32).

Epicardial approach and the diverticula neck are the helpful points toward a successful ablation in patients with CS diverticulum and posteroseptal APs $(29,30,32)$. The success rate of ablation in the neck of the 
diverticulum reaches $90 \%$ (2). After recording early retrograde atrial signal at the neck by the ablation catheter which representing CS activity, delivering controlled energy leads to elimination of APs footprints (29). However, successful ablation was also reported within the CS. In one patient with small diverticulum, ablation was done in the adjacent CS. Overall, it seems that neck of the diverticula is the site with highest rate of success for ablation $(2,4)$.

Posteroseptal APs are sometimes difficult to ablate due to complex anatomic arrangements (29, 39). Other than anatomic complexity, the inherent nature of diverticula with a pouch and a neck increase the intricacy of the task (39). Not only is the matter the difficulty of ablation of posteroseptal APs but also are the consequent life-threatening complications because of close proximity to the coronary arteries (39). It was reported that total occlusion of left circumflex artery during ablation inside the CS augments the risk of arrhythmias $(40,41)$. Even ventricular fibrillation following by sudden cardiac death was reported (40). In order to prevent complications such as perforation, formation of coagulation, excess heat, and right coronary artery stenosis during ablation procedure in the CS, some recommendations have been made like temperature control ablation, irrigated RF ablation, and cryoablation $(42,43)$. Low temperature RF ablation resulted in a safe and complication-free ablation (44).

Conclusion

CS diverticulum is a variant of CS. Importantly, it has been substrate of APs near epicardium. CS diverticulum with AP is a rare disorder. During an electrophysiologic study seeking for the underlying reasons of left posterior and posteroseptal APs, CS diverticulum is detected in coronary vein angiography. Treatment of choice for such patients is RF catheter ablation. The target site for ablation is typically the narrow neck of the CS diverticulum.

List of abbreviations

CS: coronary sinus; AP: accessory pathway; EPS: electrophysiologic study; RF: radiofrequency

References:

1. Saad EB, Marrouche NF, Cole CR, Natale A. Simultaneous Epicardial and Endocardial Mapping of a LeftSided Posteroseptal Accessory Pathway Associated with a Large Coronary Sinus Diverticulum: Successful Ablation by Transection of the Diverticulum's Neck. Pacing and clinical electrophysiology. 2002;25(10):15246 .

2. Payami B, Shafiee A, Shahrzad M, Kazemisaeed A, Davoodi G, Yaminisharif A. Posteroseptal accessory pathway in association with coronary sinus diverticulum: electrocardiographic description and result of catheter ablation. Journal of Interventional Cardiac Electrophysiology. 2013;38(1):43-9.

3. Shah SS, Teague SD, Lu JC, Dorfman AL, Kazerooni EA, Agarwal PP. Imaging of the coronary sinus: normal anatomy and congenital abnormalities. Radiographics. 2012;32(4):991-1008.

4. Selvaraj RJ, Sarin K, Singh VR, Satheesh S, Pillai AA, Kumar M, et al. Radiofrequency ablation of posteroseptal accessory pathways associated with coronary sinus diverticula. Journal of Interventional Cardiac Electrophysiology. 2016;47(2):253-9.

5. Ortale JR, Gabriel EA, Iost C, Marquez CQ. The anatomy of the coronary sinus and its tributaries. Surgical and Radiologic Anatomy. 2001;23(1):15-21.

6. Ahmed N, Perveen S, Mehmood A, Rani GF, Molon G. Coronary Sinus Ablation Is a Key Player Substrate in Recurrence of Persistent Atrial Fibrillation. Cardiology. 2019;143(3-4):107-13.

7. Chauvin M, Shah DC, Haissaguerre M, Marcellin L, Brechenmacher C. The anatomic basis of connections between the coronary sinus musculature and the left atrium in humans. Circulation. 2000;101(6):647-52.

8. Haissaguerre M, Hocini M, Takahashi Y, O'Neill MD, Pernat A, Sanders P, et al. Impact of catheter ablation of the coronary sinus on paroxysmal or persistent atrial fibrillation. J Cardiovasc Electrophysiol. 
2007;18(4):378-86.

9. Haissaguerre M, Sanders P, Hocini M, Takahashi Y, Rotter M, Sacher F, et al. Catheter ablation of long-lasting persistent atrial fibrillation: critical structures for termination. J Cardiovasc Electrophysiol. 2005;16(11):1125-37.

10. Knecht S, O'Neill MD, Matsuo S, Lim KT, Arantes L, Derval N, et al. Focal arrhythmia confined within the coronary sinus and maintaining atrial fibrillation. J Cardiovasc Electrophysiol. 2007;18(11):1140-6.

11. Sanchez-Quintana D, Lopez-Minguez JR, Pizarro G, Murillo M, Cabrera JA. Triggers and anatomical substrates in the genesis and perpetuation of atrial fibrillation. Current cardiology reviews. 2012;8(4):310-26.

12. January CT, Wann LS, Alpert JS, Calkins H, Cigarroa JE, Cleveland JC, Jr., et al. 2014 AHA/ACC/HRS guideline for the management of patients with atrial fibrillation: a report of the American College of Cardiology/American Heart Association Task Force on Practice Guidelines and the Heart Rhythm Society. Journal of the American College of Cardiology. 2014;64(21):e1-76.

13. Ho SY, Sanchez-Quintana D, Becker AE. A review of the coronary venous system: a road less travelled. Heart rhythm. 2004;1(1):107-12.

14. Nademanee K, McKenzie J, Kosar E, Schwab M, Sunsaneewitayakul B, Vasavakul T, et al. A new approach for catheter ablation of atrial fibrillation: mapping of the electrophysiologic substrate. Journal of the American College of Cardiology. 2004;43(11):2044-53.

15. Oral H, Chugh A, Good E, Wimmer A, Dey S, Gadeela N, et al. Radiofrequency catheter ablation of chronic atrial fibrillation guided by complex electrograms. Circulation. 2007;115(20):2606-12.

16. Kugler S, Nagy N, Racz G, Tokes AM, Dorogi B, Nemeskeri A. Presence of cardiomyocytes exhibiting Purkinje-type morphology and prominent connexin 45 immunoreactivity in the myocardial sleeves of cardiac veins. Heart rhythm. 2018;15(2):258-64.

17. Romero J, Gianni C, Di Biase L, Natale A. Catheter Ablation for Long-Standing Persistent Atrial Fibrillation. Methodist DeBakey cardiovascular journal. 2015;11(2):87-93.

18. Di Biase L, Santangeli P, Natale A. How to ablate long-standing persistent atrial fibrillation? Current opinion in cardiology. 2013;28(1):26-35.

19. Echeverri D, Cabrales J, Jimenez A. Myocardial venous drainage: from anatomy to clinical use. The Journal of invasive cardiology. 2013;25(2):98-105.

20. Jha NK, Gogna A, Tan TH, Wong KY, Shankar S. Atresia of coronary sinus ostium with retrograde drainage via persistent left superior vena cava. The Annals of Thoracic Surgery. 2003;76(6):2091-2.

21. Yokota M, Kyoku I, Kitano M, Shimada I, Mizuhara H, Sakamoto K, et al. Atresia of the coronary sinus orifice. Fatal outcome after intraoperative division of the drainage left superior vena cava. J Thorac Cardiovasc Surg. 1989;98(1):30-2.

22. Funabashi N, Asano M, Komuro I. Giant coronary sinus diverticulum with persistent left superior vena cava demonstrated by multislice computed tomography. International journal of cardiology. 2006;111(3):4689 .

23. Mandegar MH, Saidi B, Oraii S, Tehrai M, Roshanali F. Giant diverticulum causing ventricular arrhythmia. Journal of cardiac surgery. 2011;26(1):117-8.

24. Guiraudon GM, Guiraudon CM, Klein GJ, Sharma AD, Yee R. The coronary sinus diverticulum: a pathologic entity associated with the Wolff-Parkinson-White syndrome. American Journal of Cardiology. 1988;62(10):733-5. 
25. Isaacs D, Hazany S, Gamst A, Stark P, Mahmud E. Evaluation of the coronary sinus on chest computed tomography in patients with and without pulmonary artery hypertension. Journal of computer assisted tomography. 2009;33(4):513-6.

26. Saremi F, Krishnan S. Cardiac Conduction System: Anatomic Landmarks Relevant to Interventional Electrophysiologic Techniques Demonstrated with 64-Detector CT. RadioGraphics. 2007;27(6):1539-65.

27. Haghjoo M, Kharazi A, Fazelifar AF, Alizadeh A, Emkanjoo Z, Sadr-Ameli MA. Electrocardiographic and electrophysiologic characteristics of anteroseptal, midseptal, and posteroseptal accessory pathways. Heart rhythm. 2007;4(11):1411-9.

28. Mulder HJGH, Schalij MJ, Kauer B, Visser RF, van Dijkman PRM, Jukema JW, et al. Pravastatin and endothelium dependent vasomotion after coronary angioplasty: the PREFACE trial. Heart. 2001;86(5):533.

29. Jang SW, Rho TH, Kim DB, Kwon BJ, Cho EJ, Shin WS, et al. Successful radiofrequency catheter ablation for wolff-Parkinson-white syndrome within the neck of a coronary sinus diverticulum. Korean circulation journal. 2009;39(9):389-91.

30. Sun Y, Arruda M, Otomo K, Beckman K, Nakagawa H, Calame J, et al. Coronary sinus-ventricular accessory connections producing posteroseptal and left posterior accessory pathways: incidence and electrophysiological identification. Circulation. 2002;106(11):1362-7.

31. Saremi F, Muresian H, Sanchez-Quintana D. Coronary Veins: Comprehensive CT-Anatomic Classification and Review of Variants and Clinical Implications. RadioGraphics. 2012;32(1):E1-E32.

32. ARRUDA MS, McCLELLAND JH, WANG X, BECKMAN KJ, WIDMAN LE, GONZALEZ MD, et al. Development and Validation of an ECG Algorithm for Identifying Accessory Pathway Ablation Site in Wolff-Parkinson-White Syndrome. Journal of Cardiovascular Electrophysiology. 1998;9(1):2-12.

33. Blank R, Dieterle T, Osswald S, Sticherling C. Images in cardiovascular medicine. Wolff-ParkinsonWhite syndrome and atrial fibrillation in a patient with a coronary sinus diverticulum. Circulation. 2007;115(20):e469-71.

34. Gaita F, Paperini L, Riccardi R, Ferraro A. Cryothermic ablation within the coronary sinus of an epicardial posterolateral pathway. J Cardiovasc Electrophysiol. 2002;13(11):1160-3.

35. Traykov VB. Mapping strategies in focal atrial tachycardias demonstrating early septal activation: distinguishing left from right. Current cardiology reviews. 2015;11(2):111-7.

36. Traykov VB, Pap R, Shalganov TN, Bencsik G, Makai A, Gallardo R, et al. Electrogram analysis at the His bundle region and the proximal coronary sinus as a tool to predict left atrial origin of focal atrial tachycardias. Europace : European pacing, arrhythmias, and cardiac electrophysiology : journal of the working groups on cardiac pacing, arrhythmias, and cardiac cellular electrophysiology of the European Society of Cardiology. 2011;13(7):1022-7.

37. Saad EB, Marrouche NF, Cole CR, Natale A. Simultaneous epicardial and endocardial mapping of a left-sided posteroseptal accessory pathway associated with a large coronary sinus diverticulum: successful ablation by transection of the diverticulum's neck. Pacing and clinical electrophysiology : PACE. 2002;25(10):1524-6.

38. Justaniah A, McKee B, Silver J, Wald C, Flacke S. Coronary sinus to left atrium communication. Journal of radiology case reports. 2013;7(12):16-20.

39. Morin DP, Parker H, Khatib S, Dinshaw H. Computed tomography of a coronary sinus diverticulum associated with Wolff-Parkinson-White syndrome. Heart rhythm. 2012;9(8):1338-9.

40. Makimoto H, Zhang Q, Tilz RR, Wissner E, Cuneo A, Kuck KH, et al. Aborted sudden cardiac death due to radiofrequency ablation within the coronary sinus and subsequent total occlusion of the circumflex artery. J Cardiovasc Electrophysiol. 2013;24(8):929-32. 
41. Takahashi Y, Jais P, Hocini M, Sanders P, Rotter M, Rostock T, et al. Acute occlusion of the left circumflex coronary artery during mitral isthmus linear ablation. J Cardiovasc Electrophysiol. 2005;16(10):1104-7.

42. Hillegass WB, Patel MR, Klein LW, Gurm HS, Brennan JM, Anstrom KJ, et al. Long-term outcomes of older diabetic patients after percutaneous coronary stenting in the United States: a report from the National Cardiovascular Data Registry, 2004 to 2008. Journal of the American College of Cardiology. 2012;60(22):2280-9.

43. Veloor HP, Lokhandwala Y. A 2-year-old child with coronary sinus diverticulum and Wolff-ParkinsonWhite syndrome. Cardiol Young. 2013;23(2):274-6.

44. Fujita S, Usuda K, Hatasaki K. Catheter ablation for Wolff-Parkinson-White syndrome with coronary sinus diverticulum in a 15-year-old boy. Journal of cardiology cases. 2016;13(5):158-61.
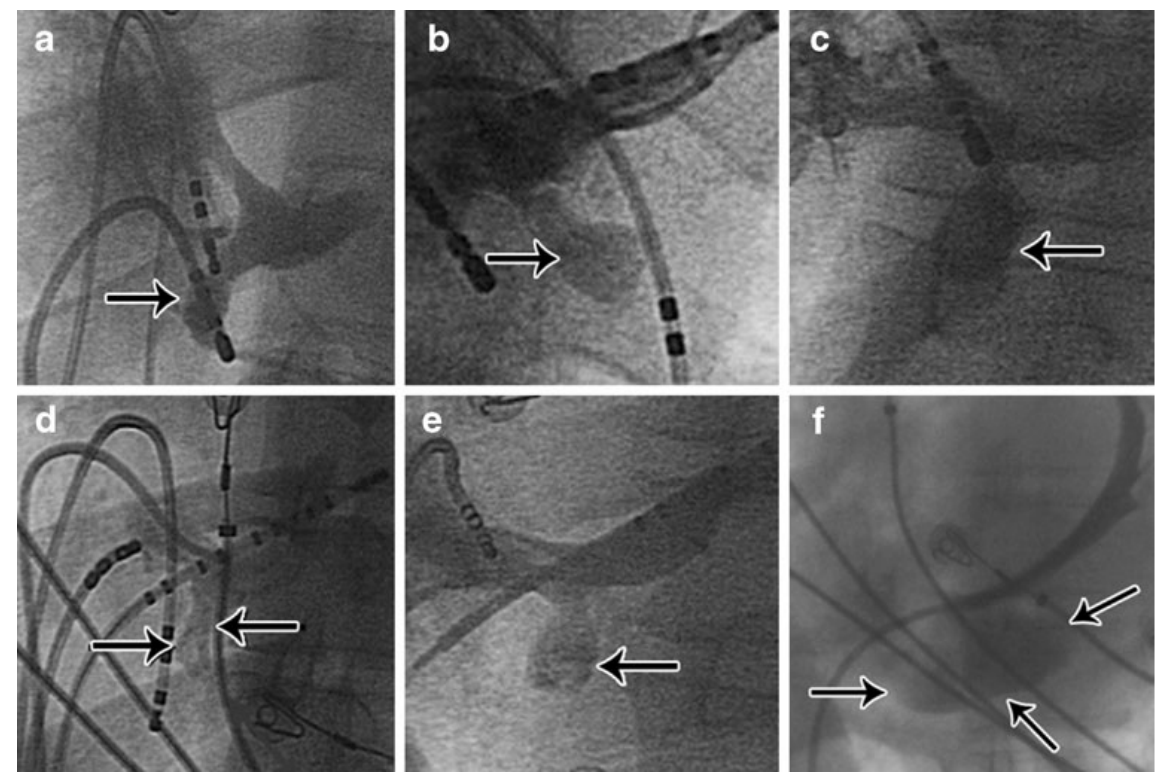

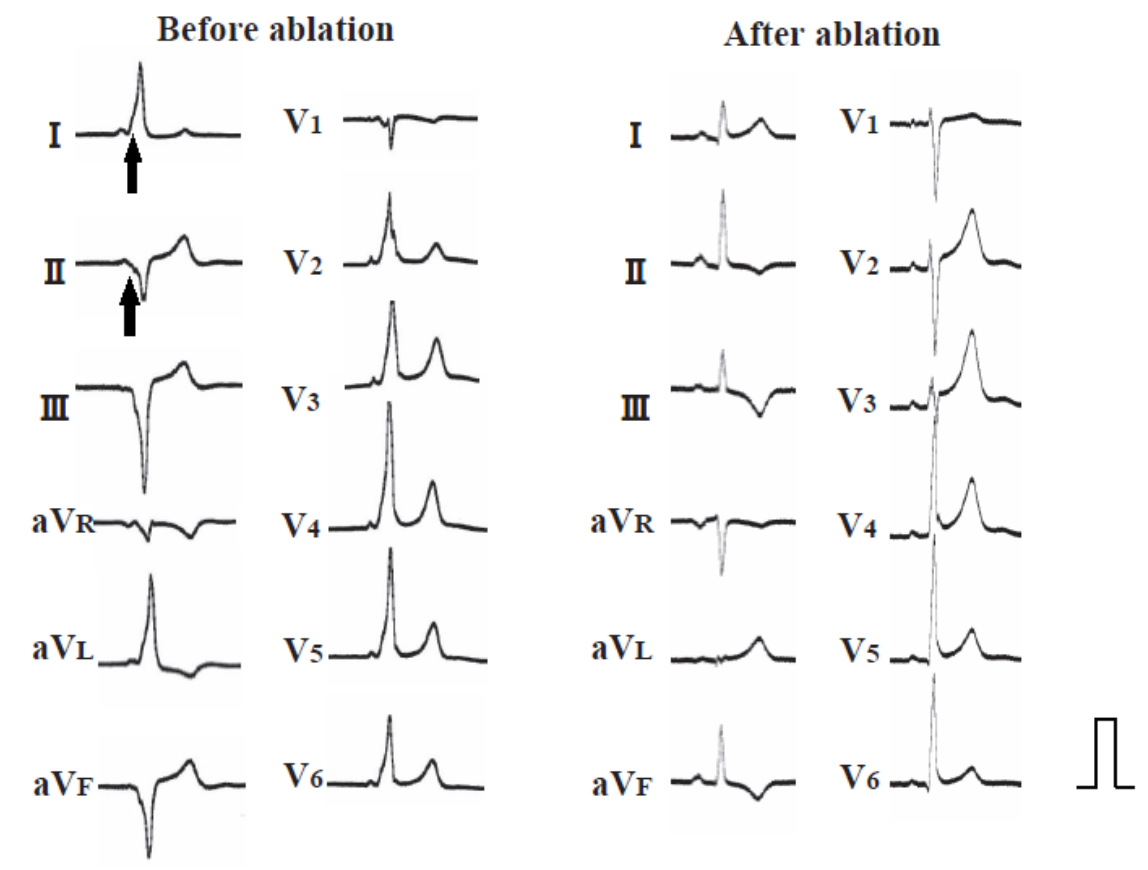